\title{
Reduced thymic output, cell cycle abnormalities, and increased apoptosis of $T$ lymphocytes in patients with cartilage-hair hypoplasia
}

\author{
Miguel A. de la Fuente, MD, PhD, ${ }^{a *}$ Mike Recher, MD, ${ }^{a *}$ Nicholas L. Rider, DO, ${ }^{c}$ Kevin A. Strauss, MD, ${ }^{c, h}$

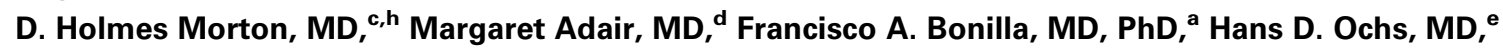

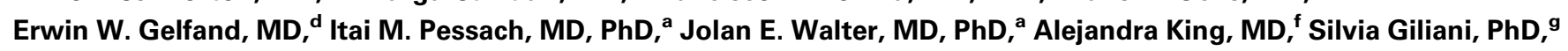 \\ Sung-Yun Pai, MD, ${ }^{\mathbf{b}}$ and Luigi D. Notarangelo, MD ${ }^{\mathbf{a}}$ Boston, Mass, Strasburg and Lancaster, Pa, Denver, Colo, Seattle, Wash, \\ Santiago, Chile, and Brescia, Italy
}

Background: Cartilage-hair hypoplasia (CHH) is characterized by metaphyseal dysplasia, bone marrow failure, increased risk of malignancies, and a variable degree of immunodeficiency. $\mathrm{CHH}$ is caused by mutations in the RNA component of the mitochondrial RNA processing $(R M R P)$ endoribonuclease gene, which is involved in ribosomal assembly, telomere function, and cell cycle control. Objectives: We aimed to define thymic output and characterize immune function in a cohort of patients with molecularly defined CHH with and without associated clinical immunodeficiency.

Methods: We studied the distribution of $B$ and $T$ lymphocytes (including recent thymic emigrants), in vitro lymphocyte proliferation, cell cycle, and apoptosis in 18 patients with $\mathrm{CHH}$ compared with controls.

Results: Patients with $\mathrm{CHH}$ have a markedly reduced number of recent thymic emigrants, and their peripheral $T$ cells show defects in cell cycle control and display increased apoptosis, resulting in poor proliferation on activation.

Conclusion: These data confirm that RMRP mutations result in significant defects of cell-mediated immunity and provide a link

From ${ }^{a}$ the Division of Immunology and the Manton Center for Orphan Disease Research and 'the Division of Hematology, Children's Hospital Boston; 'the Clinic for Special Children, Strasburg; ${ }^{d}$ the Department of Pediatrics, National Jewish Health, Denver; e the Department of Pediatrics, University of Washington School of Medicine and Seattle Children's Research Institute; ${ }^{\mathrm{f}}$ the Immunology Unit, Hospital Luis Calvo Mackenna, Santiago; " "Angelo Nocivelli" Institute for Molecular Medicine and Department of Pediatrics, University of Brescia; and ${ }^{\mathrm{h}}$ the Department of Biology, Franklin and Marshall College, Lancaster.

*These authors contributed equally to this work.

Supported by the Manton Foundation. M. Recher was supported by the Swiss National Science Foundation (SSMB; grant PASMP3-127678/1). M. Adair was supported by a fellowship from Talecris Biotherapeutics.

Disclosure of potential conflict of interest: F. A. Bonilla receives research support from Talecris Biotherapeutics, has consultant arrangements with ENTRA Pharmaceuticals and CSL Behring, receives royalties from UpToDate, and is an advisor for the Immune Deficiency Foundation. H. D. Ochs has served on the advisory board for Baxter and receives research support from the NIH, the Jeffrey Modell Foundation, and CSL Behring. The rest of the authors have declared that they have no conflict of interest.

Received for publication November 17, 2010; revised February 21, 2011; accepted for publication March 17, 2011.

Available online May 13, 2011

Reprint requests: Luigi D. Notarangelo, MD, Division of Immunology, Children's Hospital Boston, Karp Research Building, Room 9210, 1 Blackfan Circle, Boston, MA 02115. E-mail: luigi.notarangelo@ childrens.harvard.edu.

0091-6749/\$36.00

(C) 2011 American Academy of Allergy, Asthma \& Immunology

doi:10.1016/j.jaci.2011.03.042 between the cellular phenotype and the immunodeficiency in CHH. (J Allergy Clin Immunol 2011;128:139-46.)

Key words: Cartilage-hair hypoplasia, RMRP, immunodeficiency, T lymphocytes, thymus, cell cycle, apoptosis

Cartilage-hair hypoplasia $(\mathrm{CHH})$ is a rare autosomal recessive syndrome, characterized by metaphyseal dysplasia, hypoplastic hair, bone marrow failure, immunodeficiency, and an increased risk of Hirschsprung disease and malignancies. ${ }^{1,2}$ A variable degree of immunodeficiency has been reported in $\mathrm{CHH}$ that predominantly affects T-lymphocyte number and function. A minority of patients with $\mathrm{CHH}$ present with combined immunodeficiency. ${ }^{3,4}$ Hematopoietic cell transplantation is the treatment of choice in these cases. ${ }^{5,6}$ Recently, a case of antibody deficiency resembling common variable immune deficiency has been identified in an adult patient with $\mathrm{CHH}^{7}$

Cartilage-hair hypoplasia is caused by mutations of the $R M R P$ gene, ${ }^{8}$ which encodes for the RNA component of the mitochondrial RNA processing (RMRP) endoribonuclease complex that cleaves the precursor ribosomal RNA and contributes to ribosome biogenesis. ${ }^{9}$ In yeast, RMRP regulates cell cycle. $R M R P$ mutations in human beings result in decreased levels of cyclin B2 mRNA $^{10}$ and may result in chromosomal instability. Finally, recent data indicate that RMRP also forms a complex with the telomerase reverse transcriptase catalytic subunit, ${ }^{11}$ raising the possibility that telomere dysfunction is part of the cellular phenotype of the disease.

The cellular mechanisms underlying the immunodeficiency of $\mathrm{CHH}$ have remained poorly characterized, also because of the lack of animal models. It has been demonstrated that $\mathrm{T}$ cells show reduced secretion of IL- 2 and IFN- $\gamma$ after activation in vitro and enhanced apoptosis, associated with increased expression of proapoptotic molecules. ${ }^{12,13}$ Furthermore, severe abnormalities of thymic architecture have been described in patients with combined immunodeficiency (CID) caused by RMRP mutations ${ }^{4,14}$; however, no data are available on thymic function in patients with $\mathrm{CHH}$ who do not have clinical features of immunodeficiency.

Here, we present data on recent thymic emigrants (RTEs), in vitro T-lymphocyte proliferation, cell cycle, and apoptosis in 18 patients with typical features of $\mathrm{CHH}$, well defined $R M R P$ mutations, and a variable degree of immunodeficiency. Our data indicate that lymphocyte abnormalities are an integral component of $\mathrm{CHH}$, reflecting the role of RMRP in cell metabolism and function. 


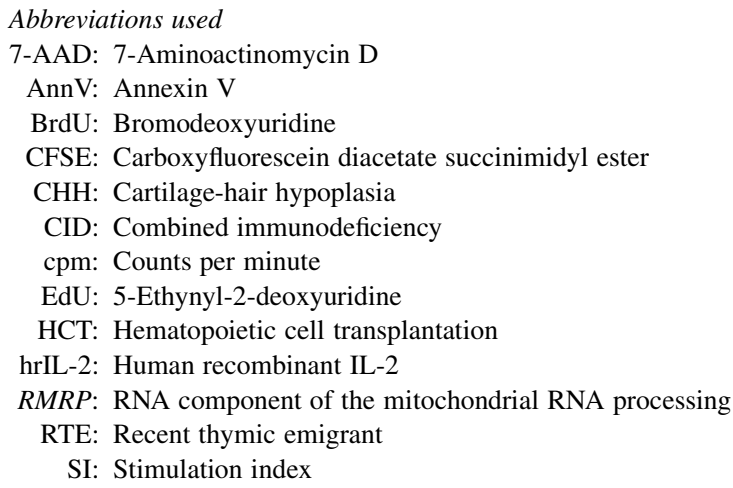

\section{METHODS \\ Patients}

Eighteen subjects with typical features of $\mathrm{CHH}$ (skeletal and hair abnormalities) were included in the study (mean age, 10.9 years; range, 1.0-21.0 years). Of these, 13 belonged to the Amish population in Pennsylvania. Deidentified information on clinical history was obtained for all patients from the referring physicians. Blood was collected from patients and controls by venipuncture. Informed consent was obtained from patients and parents in accordance with the local Institutional Review Board at Hershey Medical Center and Children's Hospital Boston, Mass.

A 21-year-old patient was included in the group 15 to 18 years old because no normal values were available for ages $>18$ years. ${ }^{15}$

Patients were classified into different clinical subsets as shown in Table I: (1) $\mathrm{CHH}$ without a history of infections, (2) $\mathrm{CHH}$ with infections, and (3) $\mathrm{CHH}$ with CID.

All patients had a confirmed mutation in the RMRP gene. For this purpose, genomic DNA was extracted from blood samples in EDTA by using standard procedures and was analyzed by PCR amplification and direct sequencing of the $R M R P$ gene as previously described. ${ }^{3}$ In the case of patient 18 , who was a compound heterozygote for a genomic insertion, the PCR fragments were cloned into a TOPO-TA cloning vector (Invitrogen, Paisley, United Kingdom). At least 10 single colonies were picked and sequenced.

\section{Fluorescence-activated cell sorting analysis of lymphocyte subpopulations}

PBMCs were separated by Ficoll gradient (Ficoll Histopaque 1077; Sigma-Aldrich, St Louis, Mo), counted, and stained with combinations of the following mAbs: CD3-APC/CD19-PERCP-CY5.5/IgD-fluorescein isothiocyanate/CD27-phycoerythrin, CD4 PERCPCY5.5/CD45RA fluorescein isothiocyanate/CD8 APC/CD31 phycoerythrin (all from BD Biosciences, San Jose, Calif). Analysis of lymphocyte subsets was performed by 4-color flow cytometry using FACS Calibur (BD Biosciences). Data were analyzed by using FlowJo software (Tree Star, Ashland, Ore).

\section{Lymphocyte proliferation}

PBMCs were labeled with carboxyfluorescein diacetate succinimidyl ester (CFSE; Molecular Probes, Eugene, Ore), $5 \mu \mathrm{mol} / \mathrm{L}$ final concentration in PBS $+0.1 \%$ BSA for 5 minutes at 37 degrees. Cells were then washed twice with RPMI 10\% FCS and cultured for 72 hours in the absence or presence of $100 \mathrm{ng} / \mathrm{mL}$ anti-CD3 (clone OKT3; eBioscience, San Diego, Calif) with or without $20 \mathrm{ng} / \mathrm{mL}$ human recombinant IL-2 (hrIL-2; Roche Applied Bioscience, Mannheim, Germany). Fluorescence-activated cell sorting analysis was performed by 4-color analysis on a FACS Calibur (BD Biosciences). Proliferation to PHA was assessed by culturing PBMCs with PHA $(10 \mu \mathrm{g} /$ $\mathrm{mL}$ final concentration) for 72 hours, followed by measuring tritiated thymidine $\left({ }^{3}[\mathrm{H}] \mathrm{TdR}\right)$ incorporation as counts per minute (cpm). Results were expressed as the stimulation index (SI), as follows:

$$
S I=[\operatorname{cpm}(\text { PHA })]-[\operatorname{cpm}(\text { medium })] /[\text { cpm }(\text { medium })] .
$$

\section{Cell cycle and cell death analysis}

PBMCs (prepared and stimulated for 96 hours as described with anti-CD3, with or without IL-2) were labeled with bromodeoxyuridine (BrdU; BD Biosciences) or with 5-ethynyl-2-deoxyuridine (EdU Click-It kit; Invitrogen, Carlsbad, Calif), according to the manufacturers' instructions.

For analysis of cell death, in vitro cultured PBMCs were stained for Annexin V (AnnV; eBioscience, San Diego, Calif; or BD Biosciences) and 7-aminoactinomycin D (7-AAD; eBioscience) in AnnV buffer for 30 minutes at room temperature.

Samples were analyzed by 4-color flow cytometry using a FACS Calibur (BD Biosciences).

\section{RESULTS}

Description of the clinical phenotype and genotype

We analyzed a cohort of 18 patients with clinical diagnosis of $\mathrm{CHH}$ and genetically confirmed RMRP mutations. Clinical features of a subgroup of the patients analyzed here were previously described in an Amish CHH cohort. ${ }^{16}$ Individual data on the clinical phenotypes are reported in Table I. Eleven patients had a history of severe and/or recurrent infections $(n=11)$, chronic diarrhea $(n=2)$, cytopenias $(n=3)$, and/or autoimmunity $(n=1)$. Three of these 11 patients had a clinical phenotype of CID (Table I). Seven patients did not have a significantly increased incidence of infections. All patients from the Amish community (patients 1-13) were homozygous for the common g.A70 $>$ G mutation. In addition, the g.C217> T and g.A118>G mutations (identified in patients 14 and 15 , respectively) had also been previously reported in patients with $\mathrm{CHH} .{ }^{10}$ In contrast, the g.G18>C, the g.G27>A, and the g.G61>A nucleotide changes (identified in patients 15,17 , and 18 , respectively) represent novel mutations. Moreover, in patient 18, we found a duplication located in the region between the TATA box and the transcription initiation site (-23-8dupTACTCTGTGAAGCTGAG).

\section{Analysis of lymphocyte subsets}

Analysis of the immunologic phenotype showed that patients' absolute total $\mathrm{CD}^{+}, \mathrm{CD}^{+}$, and $\mathrm{CD} 8^{+} \mathrm{T}$ lymphocyte numbers were low or at the lower range of normal compared with published control values for an age-matched healthy pediatric population ${ }^{15}$ (Fig 1; mean age, 10.9 years; range, 1.0-21.0 years). In most patients, the absolute number of circulating $\mathrm{CD} 19^{+}$B lymphocytes was at the lower range of normal, but the relative proportions of $\mathrm{IgD}^{-} \mathrm{CD} 27^{+}$switched memory $\mathrm{CD}^{+} 9^{+} \mathrm{B}$ cells and of $\mathrm{IgD}^{+} \mathrm{CD} 27^{+}$(mostly marginal zone-like) B cells appeared comparable to what was observed in healthy controls (Fig 1). To define whether the observed T-cell lymphopenia was secondary to defective thymopoiesis, we evaluated the absolute and relative number of $\mathrm{CD} 4{ }^{+} \mathrm{CD} 45 \mathrm{RA}^{+} \mathrm{CD} 31^{+}$RTE cells. As shown in Fig 2, both the absolute and relative numbers of RTEs were significantly reduced in the patients with $\mathrm{CHH}$, regardless of their age, compared with published normal values. ${ }^{15}$

\section{Analysis of T-cell activation and proliferation in vitro}

To define whether the T-cell lymphopenia observed in patients with $\mathrm{CHH}$ might also reflect impaired cell proliferation, we 
TABLE I. Clinical and molecular features of the patients

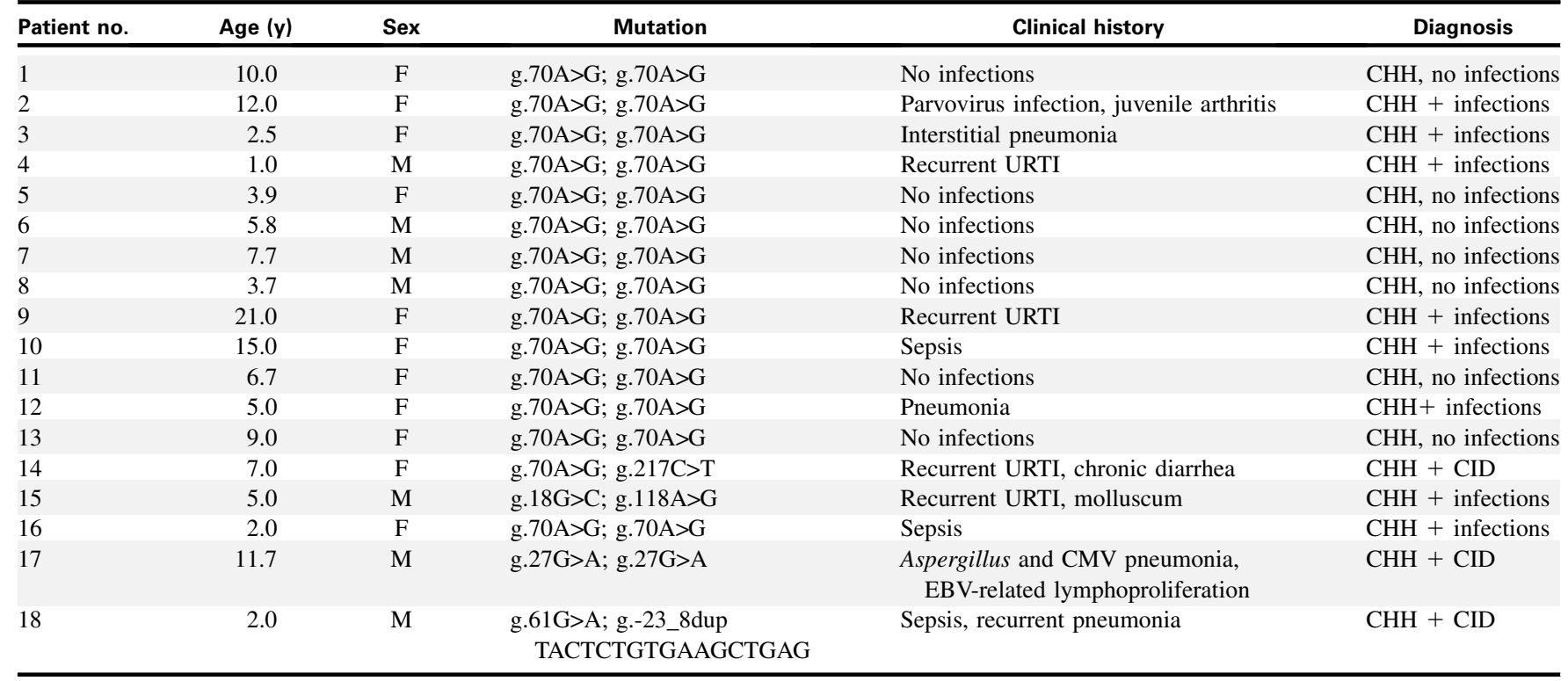

$C M V$, Cytomegalovirus; $F$, female; $M$, male; URTI, upper respiratory tract infection.

CD3

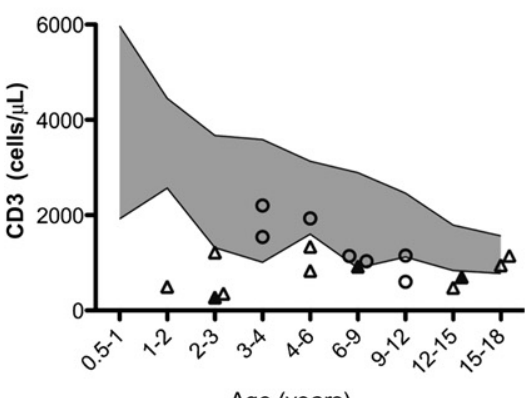

Age (years)

CD19

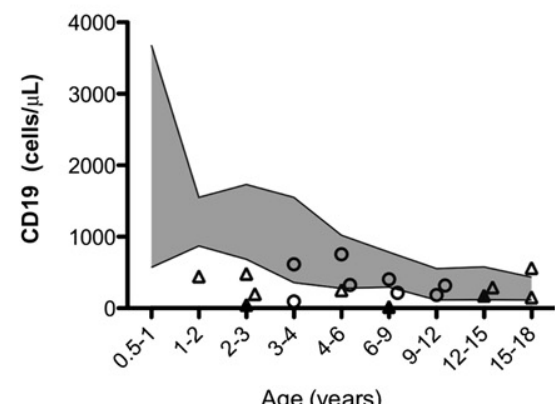

CD4

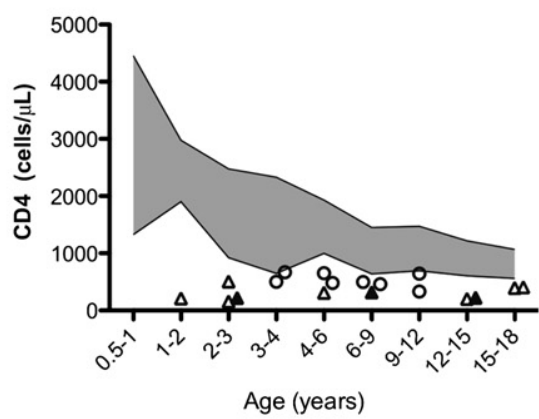

$\mathrm{CD} 19^{+} \mathrm{CD} 27^{+} \lg \mathrm{D}^{+}$

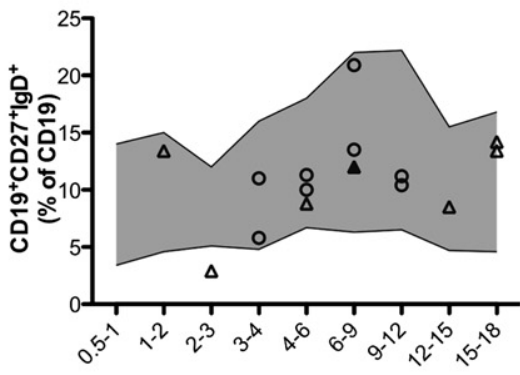

Age (years)
$\mathrm{O} \mathrm{CHH}$, no infections $\triangle \mathrm{CHH}+$ infections CD8

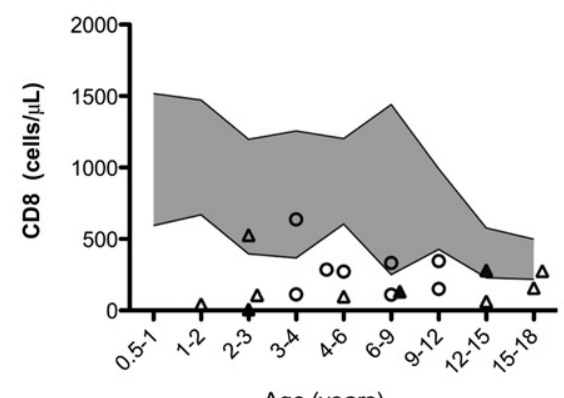

Age (years)

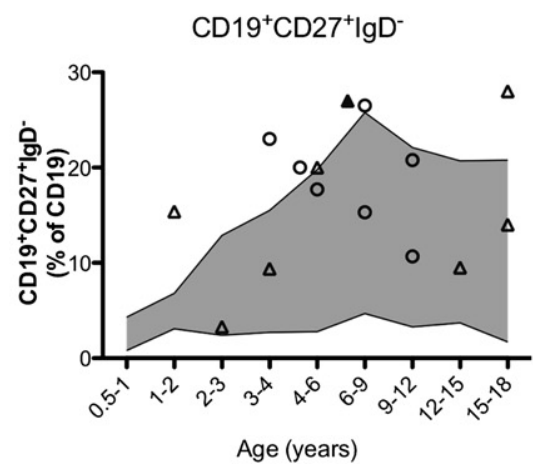

FIG 1. Lymphocyte subpopulations. In each panel, the shaded areas correspond to the value range of healthy control children from the various age groups. ${ }^{15}$

analyzed the response to PHA and anti-CD3 in vitro. As shown in Fig 3, the majority of individuals with $\mathrm{CHH}$ tested had a diminished response to PHA, with SI values that were below the lower range for normal controls $(\mathrm{SI}=60)$. PHA responses in patients with $\mathrm{CHH}$ and infection were significantly lower than in patients without infections. We also measured proliferation of
CFSE-labeled PBMCs on stimulation in vitro with anti-CD3 agonistic antibodies in the presence or absence of hrIL-2. As shown in Fig 4, proliferation of $\mathrm{CD}^{+}$lymphocytes from patients with $\mathrm{CHH}$ was impaired. In particular, under stronger (anti-CD3 ${ }^{+} \mathrm{IL}-2$ ) activating conditions, the percentage of $\mathrm{CD}^{+}$lymphocytes that did not initiate cell division was 

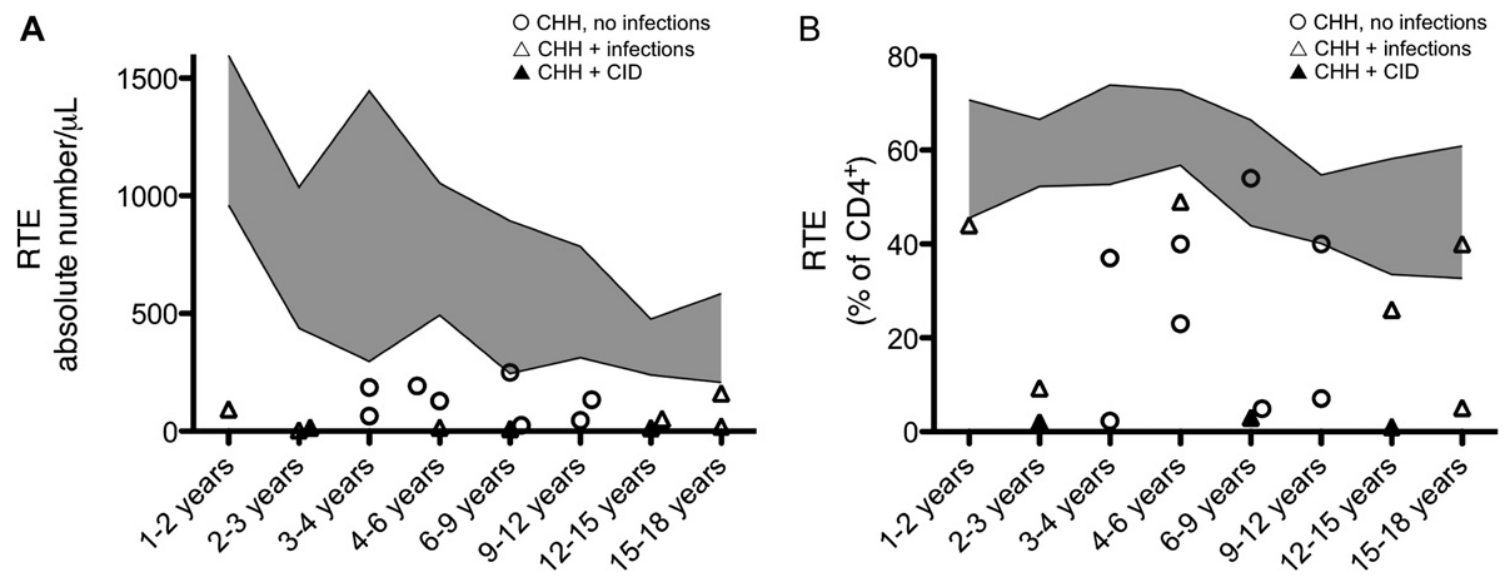

FIG 2. RTEs. The absolute (A) and relative (B) number of RTEs $\left(C D 4^{+} \mathrm{CD} 45 \mathrm{RA}{ }^{+} \mathrm{CD} 31^{+}\right)$in patients with $\mathrm{CHH}$ was plotted against the value range of healthy pediatric controls for various age groups. ${ }^{15}$

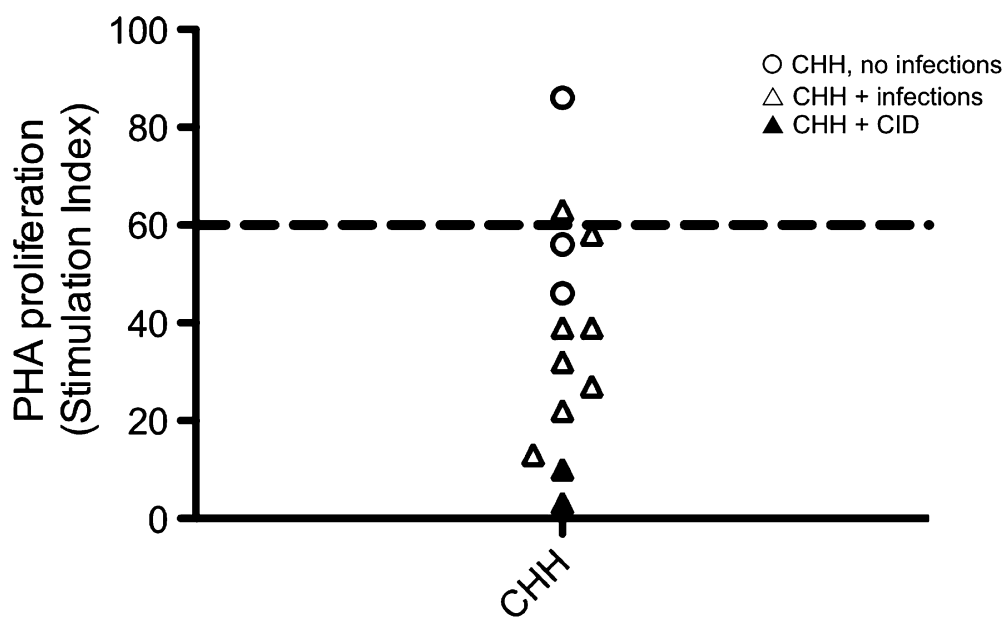

FIG 3. T-cell proliferation in response to PHA. Values are expressed as SI. The dashed line at $\mathrm{SI}=60$ represents the lower range of $\mathrm{PHA}$ response in healthy individuals.

significantly higher among patients with $\mathrm{CHH}$ (mean $\pm \mathrm{SD}$, $69.4 \pm 18.7)$ than in controls $(45.3 \pm 19.3 ; P<.05)$. Some but not absolute correlation was observed between the degree of impairment of T-cell proliferation and the severity of the clinical phenotype.

\section{Analysis of cell cycle}

RMRP has been shown to play a key role in cell cycle control. Deficiency of the RMRP homolog non-metastatic cells 1 (nme1) gene in yeast causes accumulation of cyclin B2 mRNA and delay in cell-cycle progression. ${ }^{17}$ Similar findings have been reported in fibroblasts from patients with $\mathrm{CHH}$, along with reduced levels of cyclin A2. ${ }^{10}$ We evaluated the impact of RMRP mutations on cell cycle in Tlymphocytes as a possible contributory factor to the immunodeficiency in $\mathrm{CHH}$. For this purpose, we stimulated PBMCs in vitro with agonistic anti-CD3 $\mathrm{mAb}$ (in the presence or absence of hrIL-2), followed by addition of BrdU/EdU. To assess the distribution of cells at various phases during the cell cycle, we used flow cytometry and analyzed BrdU/EdU incorporation in combination with staining for the DNA binding dye 7-AAD, which permits definition of cell ploidy. Using this method, it was possible to distinguish cells that were in $\mathrm{G} 0 / \mathrm{G} 1$ phase $\left(\mathrm{BrdU}^{-}\right.$7-AAD $\left.{ }^{\text {lo }}\right)$, synthesis $\left(\mathrm{S}\right.$ phase; $\mathrm{BrdU}^{+} 7-\mathrm{AAD}^{\mathrm{lo} / \mathrm{hi}}$ ), or $\mathrm{G} 2 / \mathrm{M}$ phase $\left(\mathrm{BrdU}^{-}\right.$ 7-AAD $\left.{ }^{\text {hi }}\right)$. As shown in Fig 5, on in vitro stimulation with anti-CD3, patients with $\mathrm{CHH}$ had an increased proportion of cells in the $\mathrm{G} 2 / \mathrm{M}$ phase (mean $\pm \mathrm{SD}, 12.8 \pm 5.7$ vs $7.4 \pm 6.7$ in patients vs controls; $P<.05$ ). An increase of cells in the $\mathrm{G} 2 / \mathrm{M}$ phase was also observed in lymphocytes of patients with $\mathrm{CHH}$ on stimulation with anti-CD3 + IL-2, although it did not reach statistical significance, and was associated with a marked decrease of lymphocytes in $\mathrm{S}$ phase (mean $\pm \mathrm{SD}, 31.1 \pm 11.2$ vs $45.1 \pm 11.1$ for patients vs controls; $P<.05)$. The reduced proportion of cells entering $S$ phase and the prolongation of the cell cycle (with a higher proportion of cells in the G2/M phase) are consistent with the reduced number of cell divisions and the defective T-cell proliferation reported.

\section{Increased activation-induced cell death in patients $\mathrm{CHH}$}

Increased T-cell apoptosis has been previously reported in 1 patient with $\mathrm{CHH}^{13}$ but has not been further investigated. We have analyzed activation-induced apoptosis in lymphocytes 


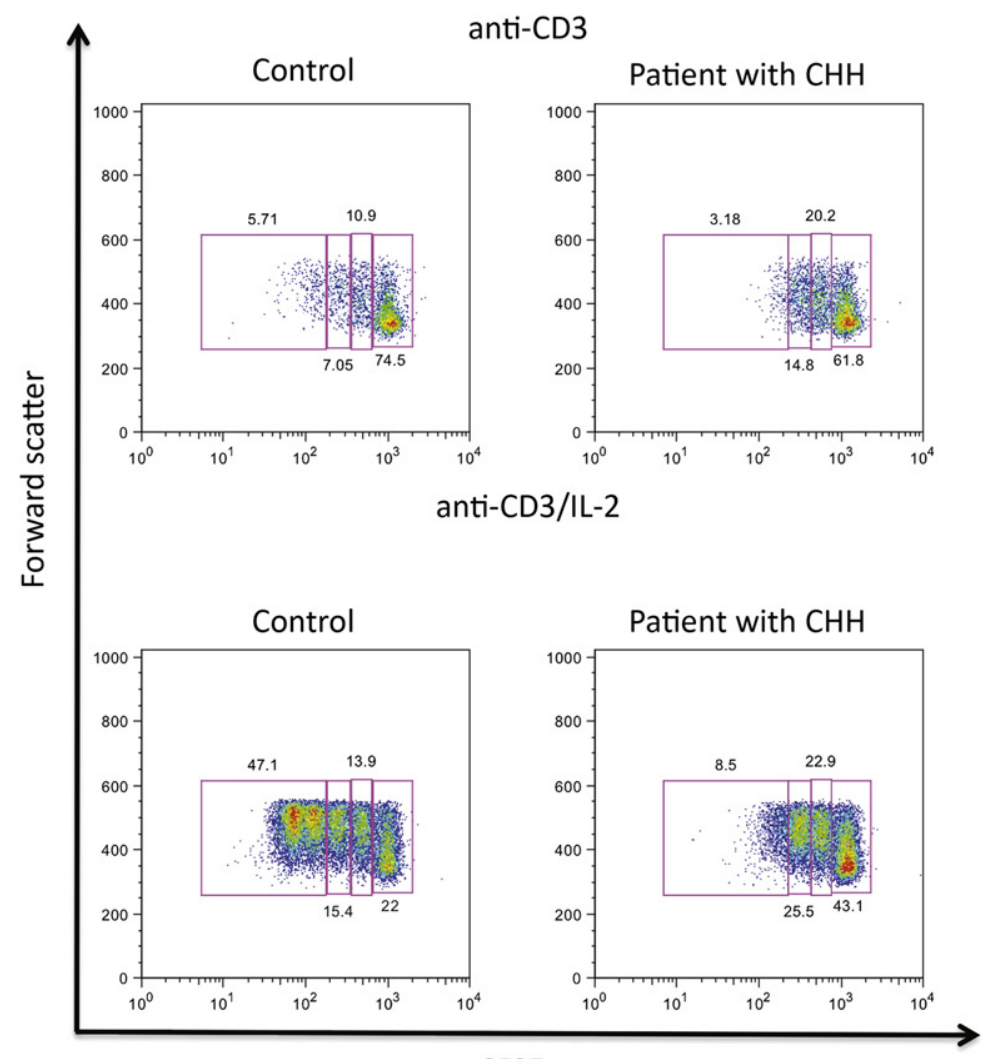

CFSE
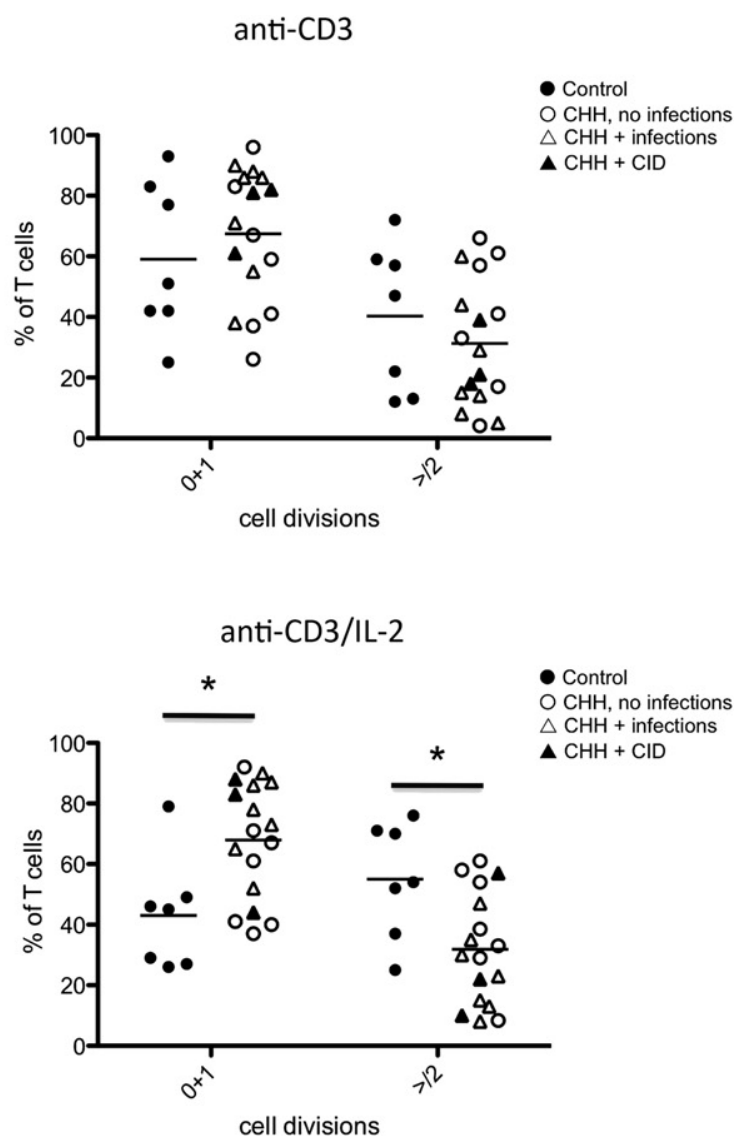

FIG 4. T-cell proliferation in response to agonistic anti-CD3 antibody. Left, Representative examples of proliferation of control-derived or patient-derived PBMCs in response to anti-CD3 (top) or anti-CD3 + IL-2 (bottom), as measured by CFSE dilution of $\mathrm{CD}^{+}$cells. Boxes identify different rounds of proliferation, and the proportion of cells within each box is annotated. Gating was done on viable cells. Right, Proportion of PBMCs undergoing 0 to 1 or $\geq 2$ rounds of cell division in response to anti-CD3 (top) or anti-CD3 + IL-2 (bottom). Individual symbols identify single subjects. In each diagram, the horizontal bar represents the mean value. ${ }^{*} P<.05$

from patients with $\mathrm{CHH}$ and controls after in vitro culture with anti-CD3 $\mathrm{mAb}$ in the presence or absence of IL-2. We used flow cytometry by simultaneously staining the cells for AnnV and for incorporation of 7-AAD. This method allows identification of viable $\left(\mathrm{AnnV}^{-}\right.$7-AAD $\left.{ }^{-}\right)$, early apoptotic $\left(\mathrm{AnnV}^{+}\right.$ 7-AAD $\left.{ }^{-}\right)$, and late apoptotic/necrotic $\left(\mathrm{AnnV}^{+}\right.$7-AAD $\left.{ }^{+}\right)$cells. As shown in Fig 6, the percentage of both early and late apoptotic cells was increased in patients with $\mathrm{CHH}$ versus healthy controls, reaching a statistically significant difference for late apoptotic/necrotic cells on culture with anti-CD3 mAb + IL-2 (mean \pm SD in patients with $\mathrm{CHH}$ vs controls, $6.5 \pm 4.6$ vs $2.1 \pm 1.4 ; P<.05$ ).

\section{Correction of T-cell proliferation after hematopoietic cell transplantation}

Patients with $\mathrm{CHH}$ and severe immunodeficiency may require hematopoietic cell transplantation (HCT), and this treatment often allows prolonged survival with immune reconstitution., We have shown that $R M R P$ mutations cause abnormal progression along the cell cycle and defective T-cell proliferation after stimulation. Because of severe infections, EBV-related lymphoproliferation, and chronic lung disease, patient 17 received HCT from his HLA-phenotypically identical father after reduced-intensity conditioning with fludarabine $150 \mathrm{mg} / \mathrm{m}^{2}$, melphalan $140 \mathrm{mg} / \mathrm{m}^{2}$, and alemtuzumab $1 \mathrm{mg} / \mathrm{kg}$. This treatment resulted in mixed/split chimerism, with $90 \%$ donor $\mathrm{T}$ cells and $1 \%$ donor myeloid cells, respectively, at 11 months after HCT. We compared the ability of the patient's T lymphocytes to undergo multiple rounds of cell division in response to in vitro stimulation with anti-CD3 + IL-2 before and after HCT (Fig 7). As expected, before HCT, most of the patient's $\mathrm{CD}^{+}{ }^{+} \mathrm{T}$ cells failed to divide or underwent a single round of proliferation; in contrast, after HCT, the majority of the cells underwent multiple rounds of cell division. These results confirm the cell-intrinsic role of $R M R P$ for normal T-lymphocyte proliferation.

\section{DISCUSSION}

Significant variability in the clinical phenotype has been reported in patients with $R M R P$ mutations. There is some evidence that the type and level of RMRP functional impairment may correlate with the severity of the clinical spectrum. In particular, mutations that affect ribosomal RNA cleavage and ribosomal assembly are associated with more severe bone dysplasia, whereas mutations that reduce mRNA cleavage have a more significant impact on cell cycle regulation and are associated 

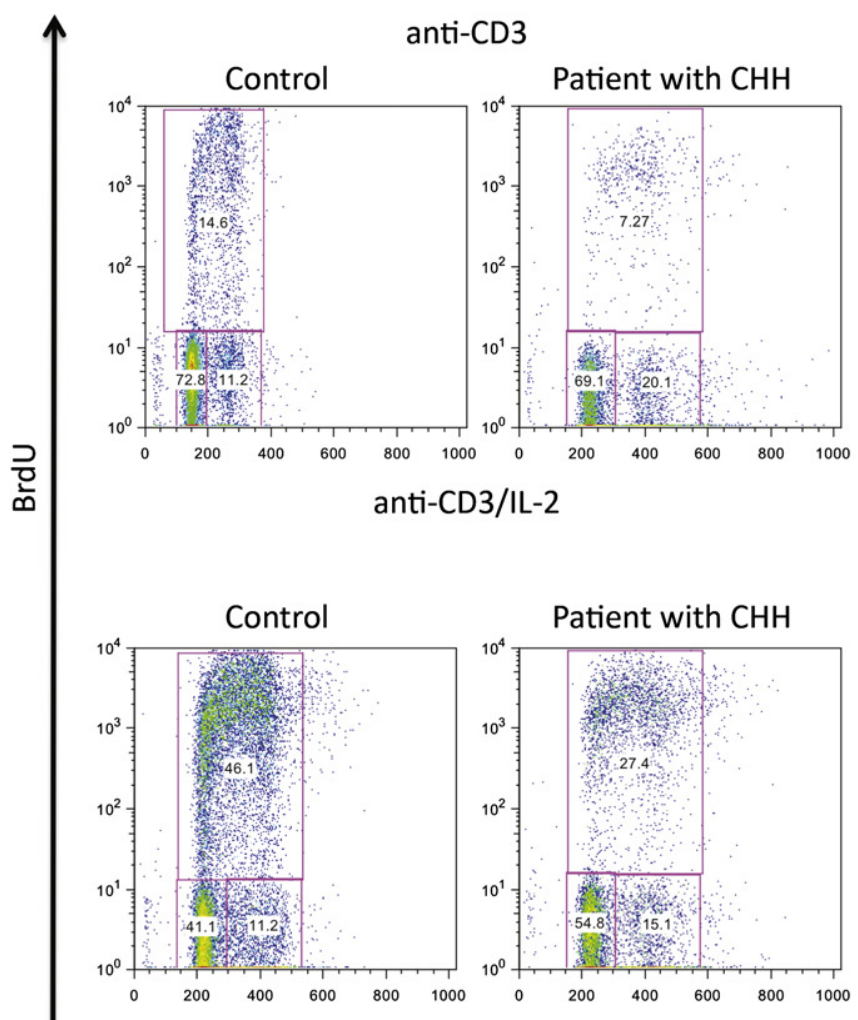

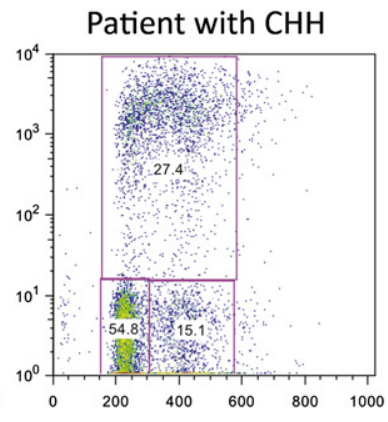

7-AAD

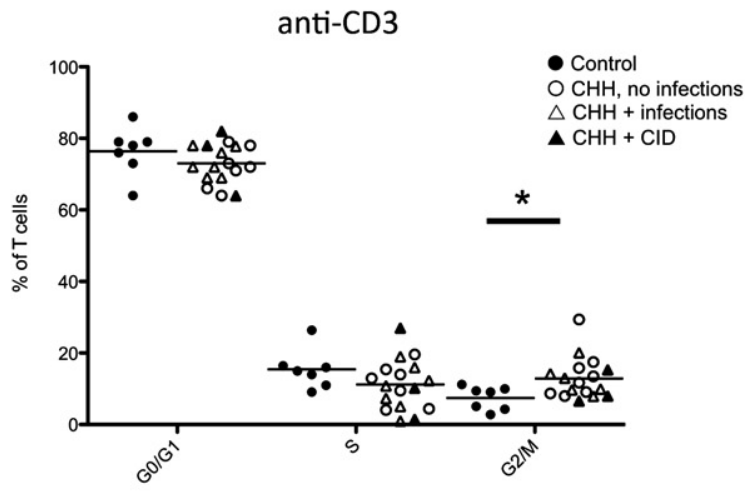

anti-CD3+IL-2

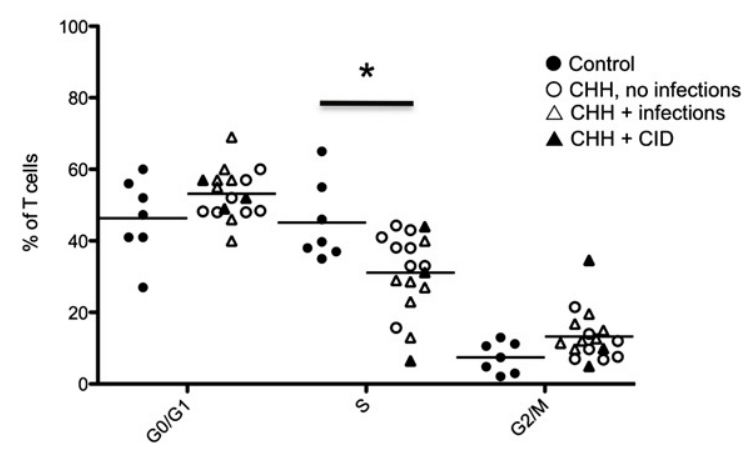

FIG 5. Cell cycle analysis. Left, Representative examples of cell cycle analysis in control-derived or patientderived PBMCs on in vitro stimulation with anti-CD3 (top) or anti-CD3 + IL-2 (bottom). Boxes identify cells in $\mathrm{G0} / \mathrm{G} 1$ (bromodeoxyuridine $[\mathrm{BrdU}]^{-}$7-AAD ${ }^{-}$), $\mathrm{S}\left(\mathrm{BrdU}^{+}\right.$7-AAD ${ }^{-/+}$), and $\mathrm{G} 2 / \mathrm{M}\left(\mathrm{BrdU}^{-}\right.$7-AAD ${ }^{+}$) phases of the cell cycle. The proportion of cells within each of these is annotated. Right, Proportion of PBMCs in G0-G1, S, and G2/M phases on in vitro culture with anti-CD3 (top) or anti-CD3 + IL-2 (bottom). Individual symbols identify single subjects. In each diagram, the horizontal bar represents the mean value. ${ }^{*} P<.05$.

with a higher incidence of immunologic and hematologic abnormalities and cancer. ${ }^{10}$ Although imprecise, this reported genotype-phenotype correlation may contribute to significant variability in the degree of immunodeficiency among patients with $\mathrm{CHH}^{2-6,16,18}$ The majority of the patients included in this study belonged to an Amish population and were homozygous for the g.A70 $>\mathrm{G}$ mutation. The observation that in vitroactivated lymphocytes from the patients' cohort showed accumulation of cells in the G2/M phase and decreased proportions of cells in the $\mathrm{S}$ phase, associated with impaired proliferation, is consistent with the demonstration that transient transfection of normal human fibroblasts with the g.A70 $>\mathrm{G}$ $R M R P$ mutant construct causes very severe impairment of cyclin B2 mRNA cleavage. ${ }^{10}$

Our data indicate that $R M R P$ mutations have a drastic effect on lymphocyte maturation and function, and that they interfere with thymic generation of T lymphocytes. This is not unexpected, because thymic generation of $\mathrm{T}$ lymphocytes requires robust proliferation of thymocyte progenitors. Consistent with a potential role of $R M R P$ in supporting thymopoiesis, we and others have reported profound abnormalities of thymic architecture in some patients with RMRP mutations. ${ }^{4,14}$ Furthermore, we previously reported low to undetectable levels of T-cell receptor excision circles (another marker to assess de novo thymic generation of
T lymphocytes) in 6 of 7 patients with $\mathrm{CHH}^{3}$; however, all 7 of these patients had significant clinical features suggestive of CID. In this article, we demonstrate that a low number of RTEs may also be seen in patients with $\mathrm{CHH}$ without significant clinical immunodeficiency. Along with defects in cell cycle progression and increased apoptosis, these data suggest that at least certain $R M R P$ mutations are consistently associated with severe cellular immunodeficiency. It will be important to perform similar studies in a larger cohort of patients with different mutations to verify whether RMRP mutations known to interfere with cyclin B2 mRNA processing and cell cycle progression consistently cause abnormalities of lymphocyte function.

Our results also imply that in contrast with T-cell maturation, B-cell maturation was less affected by RMRP mutations. B-cell function was not formally addressed in the current article; however, we collected data on serum immunoglobulins in 14 of the 18 patients with $\mathrm{CHH}$. With the exception of the patients with $\mathrm{CHH}$ and $\mathrm{CID}$, all patients with $\mathrm{CHH}$ tested had normal immunoglobulin levels. Nine of them were evaluated for tetanus-toxoid specific antibodies, and 7 had protective $(>0.15 \mathrm{IU} / \mathrm{mL})$ titers. $\mathrm{Pa}-$ tient 16 was also immunized with bacteriophage $\varnothing X 174$ and showed a clearly diminished $\mathrm{IgG}$ response suggestive of a functional $\mathrm{T}_{\mathrm{H}}$-cell defect reminiscent of patients with CD40 ligand gene $(C D 40 L G)$ mutations. 

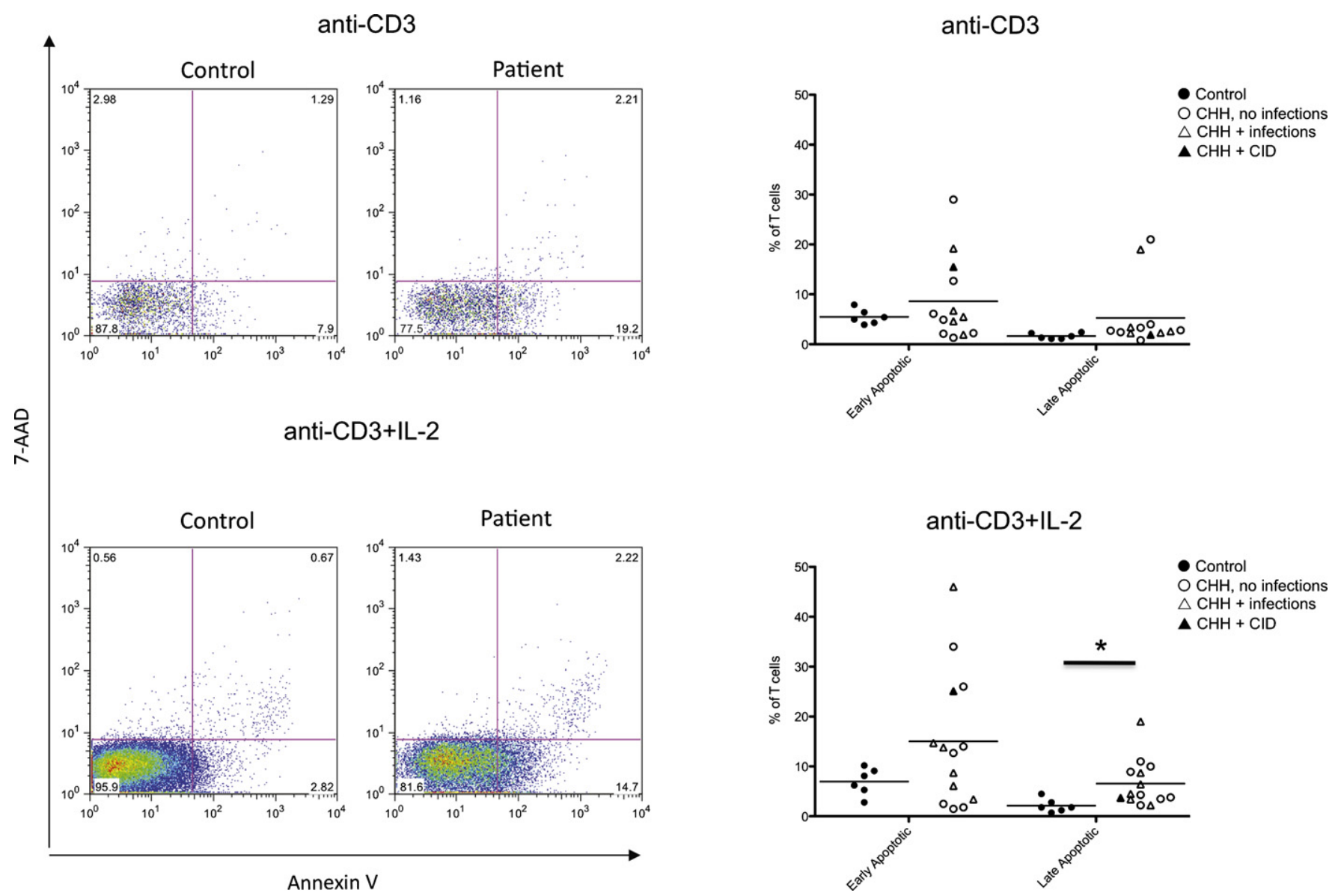

FIG 6. Analysis of apoptosis and cell death. Left, Representative example of fluorescence-activated cell sorting plots after staining of PBMCs for AnnV and 7-AAD in healthy controls and patients with $\mathrm{CHH}$ on in vitro stimulation with anti-CD3 (top) or anti-CD3 + IL-2 (bottom). Right, Proportion of early apoptotic $\left(A n n V^{+} 7-A A D^{-}\right)$and late apoptotic/necrotic $\left(\mathrm{AnnV}^{+} 7-\mathrm{AAD}^{+}\right)$PBMCs on in vitro culture with anti-CD3 (top) or anti-CD3 + IL-2 (bottom). Individual symbols identify single subjects. In each diagram, the horizontal bar represents the mean value. ${ }^{*} P<.05$.

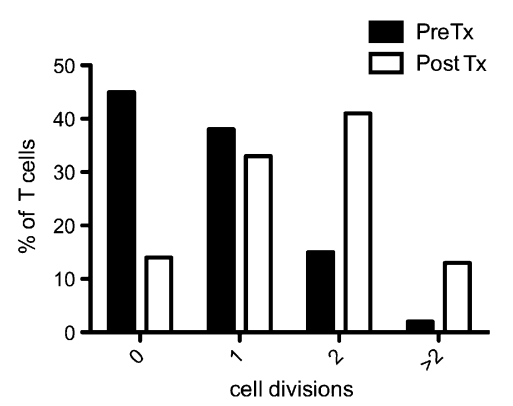

FIG 7. Correction of T-cell proliferation defect after HCT in a patient with $\mathrm{CHH}$. The proportion of T lymphocytes undergoing $0,1,2$ or $>2$ rounds of proliferation (as indicated by CFSE dilution) after 72 hours of in vitro culture with anti-CD3 + IL-2 is shown for patient 17 before (black bars) or 11 months after (white bars) HCT from his HLA phenotypically identical father. $T x$, Transplantation.

Various mechanisms may contribute to the cell cycle abnormalities and increased apoptosis observed in our series of patients. In particular, $\mathrm{CHH}$ belongs to a family of diseases, collectively called ribosomopathies, in which well defined components of the ribosome RNA/protein complex are defective. ${ }^{9}$
These diseases mostly affect the hematopoietic lineage. Evidence from animal models indicates that when ribosomal function is disturbed, the levels of master regulator p53 are increased, because the p53 ubiquitin ligase murine double minute 2 binds to free ribosomal proteins. ${ }^{19}$ Increased p53 is associated with cell cycle arrest and apoptosis. ${ }^{20}$ The possibility that p53 levels are increased in $\mathrm{CHH}$ and may contribute to the immunodeficiency remains to be investigated.

Finally, we have confirmed significant heterogeneity of the $\mathrm{CHH}$ clinical phenotype (including variable degree of immunodeficiency), even among patients who share the same $R M R P$ genetic defect. Given the large number of patients from the same Amish community, there are limitations to the genetic variability seen in the present cohort. The basis for heterogeneity of the clinical phenotype of $\mathrm{CHH}$ remains unknown, because it cannot be easily attributed to either genetic or environmental factors.

In summary, we show that in $\mathrm{CHH}$, despite individual variability, there is a clear defect in the thymic generation of $\mathrm{T}$ lymphocytes and in peripheral T-cell proliferation, cell cycle control, and activation-induced cell death. These data provide a common ground for understanding the functional basis of the underlying immunodeficiency in $\mathrm{CHH}$. 
Clinical implications: Defects of thymic function and of T-lymphocyte cell cycle control, survival, and proliferation likely contribute to the increased frequency of severe infections in patients with $\mathrm{CHH}$.

\section{REFERENCES}

1. Notarangelo LD, Roifman CM, Giliani S. Cartilage-hair hypoplasia: molecula basis and heterogeneity of the immunological phenotype. Curr Opin Allergy Clin Immunol 2008;8:534-9.

2. Mäkitie O, Kaitila I. Cartilage-hair hypoplasia-clinical manifestations in 108 Finnish patients. Eur J Pediatr 1993;152:211-7.

3. Kavadas FD, Giliani S, Gu Y, Mazzolari E, Bates A, Pegoiani E, et al. Variability of clinical and laboratory features among patients with ribonuclease mitochondrial RNA processing endoribonuclease gene mutations. J Allergy Clin Immunol 2008 122:1178-84.

4. Roifman CM, Gu Y, Cohen A. Mutations in the RNA component of RNase mitochondrial RNA processing might cause Omenn syndrome. J Allergy Clin Immunol 2006;117:897-903.

5. Guggenheim R, Somech R, Grunebaum E, Atkinson A, Roifman CM. Bone marrow transplantation for cartilage-hair-hypoplasia. Bone Marrow Transplant 2006; 38:751-6.

6. Bordon V, Gennery AR, Slatter MA, Vandecruys E, Laureys G, Veys P, et al. Clinical and immunologic outcome of patients with cartilage hair hypoplasia after hematopoietic stem cell transplantation. Blood 2010;116:27-35.

7. Horn J, Schlesier M, Warnatz K, Prasse A, Superti-Furga A, Peter HH, et al. Fatal adult-onset antibody deficiency syndrome in a patient with cartilage hair hypoplasia. Hum Immunol 2010;71:916-9.

8. Ridanpää M, van Eenennaam H, Pelin K, Chadwick R, Johnson C, Yuan B, et al Mutations in the RNA component of RNase MRP cause a pleiotropic human disease, cartilage-hair hypoplasia. Cell 2001;104:195-203.

9. Narla A, Ebert BL. Ribosomopathies: human disorders of ribosome dysfunction Blood 2010;115:196-205
10. Thiel CT, Mortier G, Kaitila I, Reis A, Rauch A. Type and level of RMRP functional impairment predicts phenotype in the cartilage hair hypoplasia-anauxetic dysplasia spectrum. Am J Hum Genet 2007;81:519-29.

11. Maida Y, Yasukawa M, Furuuchi M, Lassmann T, Possemato R, Okamoto N, et al. An RNA-dependent RNA polymerase formed by TERT and the RMRP RNA. Nature 2009;461:230-5.

12. Castigli E, Irani AM, Geha RS, Chatila T. Defective expression of early activation genes in cartilage-hair hypoplasia $(\mathrm{CHH})$ with severe combined immunodeficiency (SCID). Clin Exp Immunol 1995;102:6-10.

13. Yel L, Aggarwal S, Gupta S. Cartilage-hair hypoplasia syndrome: increased apoptosis of $\mathrm{T}$ lymphocytes is associated with altered expression of Fas (CD95), FasL (CD95L), IAP, Bax, and Bcl2. J Clin Immunol 1999;19: 428-34.

14. Poliani PL, Facchetti F, Ravanini M, Gennery AR, Villa A, Roifman CM, et al. Early defects in human T-cell development severely affect distribution and maturation of thymic stromal cells: possible implications for the pathophysiology of Omenn syndrome. Blood 2009;114:105-8.

15. van Gent R, van Tilburg CM, Nibbelke EE, Otto SA, Gaiser JF, Janssens-Korpela PL, et al. Refined characterization and reference values of the pediatric T- and B-cell compartments. Clin Immunol 2009;133:95-107.

16. Rider NL, Morton DH, Puffenberger E, Hendrickson CL, Robinson DL, Strauss KA. Immunologic and clinical features of 25 Amish patients with RMRP 70 A $\rightarrow$ G cartilage hair hypoplasia. Clin Immunol 2009;131:119-28.

17. Cai T, Aulds J, Gill T, Cerio M, Schmitt ME. The Saccharomyces cerevisiae RNase mitochondrial RNA processing is critical for cell cycle progression at the end of mitosis. Genetics 2002;161:1029-42.

18. Mäkitie O, Kaitila I, Savilahti E. Susceptibility to infections and in vitro immune functions in cartilage-hair hypoplasia. Eur J Pediatr 1998;157:816-20.

19. Fumagalli S, Di Cara A, Neb-Gulati A, Natt F, Schwemberger S, Hall J, et al. Absence of nucleolar disruption after impairment of $40 \mathrm{~S}$ ribosome biogenesis reveals an rpL11-translation-dependent mechanism of p53 induction. Nat Cell Biol 2009; 11:501-8.

20. Schwartz D, Rotter V. p53-dependent cell cycle control: response to genotoxic stress. Semin Cancer Biol 1998;8:325-36. 